\title{
CRACK PROPAGATION IN THE HUMAN BONE. MODE I OF FRACTURE
}

\author{
E.M. Craciun ${ }^{1}$, A. Rabaea $^{2}$, M.F. Popa ${ }^{1, *}$, C.I. Mihailov ${ }^{1}$
}

\begin{abstract}
The problem of crack propagation in human bone is studied. We formulate and solve the mathematical problem for the pre-stressed crack in Mode I of classical fracture. Using the boundary conditions on the crack faces in the bone, regarded as an elastic composite material, we solve our Riemann-Hilbert problem. Using generalized Sih's strain energy density generalized and maximum stress criteria we find the direction of the crack path in Iliac bone, regarded as a pre-stressed orthotropic composite.
\end{abstract}

\section{Introduction}

Fracture of bones represents a current interest in Biomechanics, [4], [16]-[17].

Bone defects, i.e. holes and cracks, constitute a frequent problem in human and veterinary medicine. Bone is a complex material, a hard connective tissue and forms rigid skeleton. It could be regarded as an anisotropic elastic composite material, [16].

In the literature, using analytical, numerical or experimental researches the modelling of composite materials and of the cracked composite materials was intensely studied in the last decades, [1]-[3], [5]-[10].

Crack initialization, critical forces that produces crack instability and crack propagation direction are most important subjects for mathematical modelling of Fracture mechanics. We consider that the admissible equilibrium states of

Key Words: crack in mode I of Fracture, anisotropic materials, initial fields, propagation criteria, Iliac bone

Received: March, 2017.

Revised: May, 2017.

Accepted: June, 2017. 
our body are plane strain states relative to $O x_{1} x_{2}$ plane. In this case the equilibrium states can be represented by two complex potentials defined in two complex planes, [8], [11]-[14].

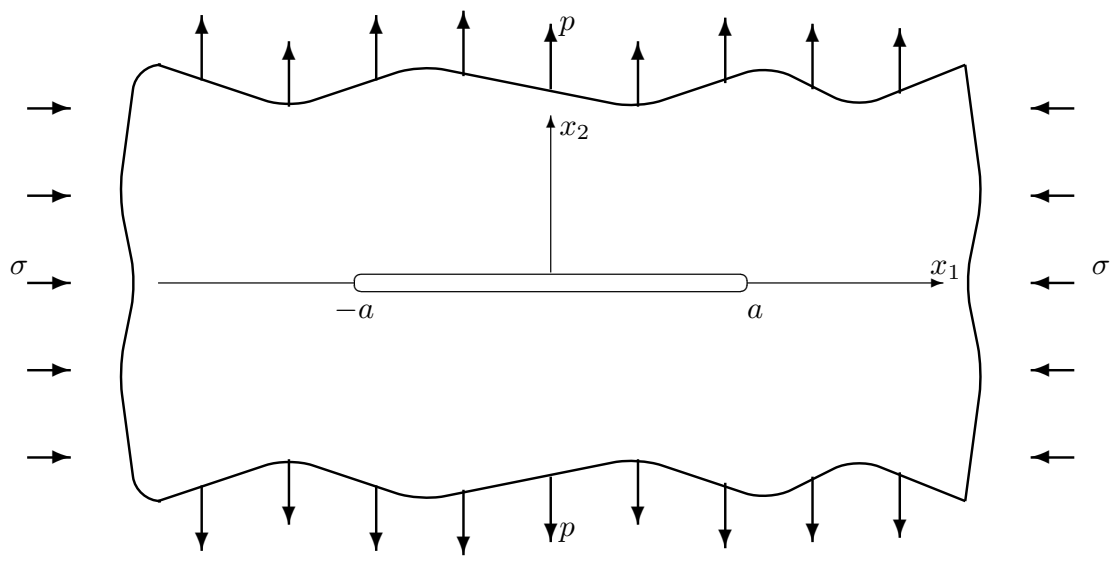

Figure 1: Pre-stressed crack acted in mode I of Fracture

Using Sih's generalized strain energy density criterion and generalized maximum tensile stress criterion we find the direction of the crack path for a prestressed crack in mode I of Fracture being in Iliac bone. Both generalized crack propagation criteria furnish us that the crack will propagate along its line.

\section{Representation of the incremental fields. Mode I of Fracture}

In this Section, following [8], [11], we present the incremental plane state for a pre-stressed homogeneous anisotropic material.

Our body is characterized by the following governing equations:

incremental equilibrium equation

$$
\operatorname{div} \boldsymbol{\vartheta}=\mathbf{0} \text { or } \vartheta_{k l, k}=0, k, l=1,2,3 \text { in } B,
$$

incremental constitutive equation

$$
\boldsymbol{\vartheta}=\boldsymbol{\omega} \boldsymbol{\nabla} \mathbf{u}^{T} \text { or } \vartheta_{k l}=\omega_{k l m n} u_{m, n}, k, l, m, n=1,2,3 .
$$

mixed incremental boundary conditions

$\mathbf{u}=\mathbf{v}$ or $u_{k}=v_{k}$ on $S_{1}$ and $\mathbf{s}_{\mathbf{n}}=\vartheta^{T} \mathbf{n}=\mathbf{l}$ or $s_{\mathbf{n} l}=\vartheta_{k l} n_{k}=l_{l}$ on $S_{2}$. 
In above equations, we denote by $\boldsymbol{\vartheta}=\boldsymbol{\vartheta}(\mathbf{x})$ and respectively by $\vartheta_{k l, k}$ incremental nominal stress tensor and the derivative of its components, respectively, by $\mathbf{u}=\mathbf{u}(\mathbf{x})$ and respectively by $u_{m, n}$ the incremental displacement field and the derivative of its components, respectively. The incremental displacement imposed on the part $S_{1}$ of the boundary $\partial B$ we denoted by $\mathbf{v}=\mathbf{v}(\mathbf{x})$ and the incremental dead traction imposed in the complementary part $S_{2}$ of the boundary by $\mathbf{l}=\mathbf{l}(\mathbf{x})$.

The expressions of the components of the instantaneous elasticity are given by

$$
\omega_{k l m n}=c_{k l m n}+\sigma_{k n} \delta_{m l},
$$

where $c_{k l m n}$ are the components of the elasticity tensor $\mathbf{c}$ of the considered linear, hyperelastic material and $\sigma_{k n}$ are the components of the Cauchy's stress tensor $\boldsymbol{\sigma}$ corresponding to the initial deformation.

In what follows, we shall assume that the material is orthotropic, the symmetry planes being the coordinate planes and using the Voigt's convention the incremental constitutive equation is:

$$
\left[\begin{array}{c}
\vartheta_{1} \\
\vartheta_{2} \\
\vartheta_{3} \\
\vartheta_{4} \\
\vartheta_{5} \\
\vartheta_{6}
\end{array}\right]=\left[\begin{array}{cccccc}
C_{11} & C_{12} & C_{13} & 0 & 0 & 0 \\
C_{12} & C_{22} & C_{23} & 0 & 0 & 0 \\
C_{13} & C_{23} & C_{33} & 0 & 0 & 0 \\
0 & 0 & 0 & C_{44} & 0 & 0 \\
0 & 0 & 0 & 0 & C_{55} & 0 \\
0 & 0 & 0 & 0 & 0 & C_{66}
\end{array}\right]\left[\begin{array}{c}
\varepsilon_{1} \\
\varepsilon_{2} \\
\varepsilon_{3} \\
\varepsilon_{4} \\
\varepsilon_{5} \\
\varepsilon_{6}
\end{array}\right] .
$$

We have the following expressions of the non-vanishing independents components of the stiffness matrix $[C]$ of an orthotropic material, as function of its engineering constants:

$$
\begin{aligned}
& C_{11}=\frac{1-\nu_{23} \nu_{32}}{E_{2} E_{3} \Delta}, C_{12}=\frac{\nu_{21}+\nu_{31} \nu_{23}}{E_{2} E_{3} \Delta}=\frac{\nu_{12}+\nu_{32} \nu_{13}}{E_{1} E_{3} \Delta}, \\
& C_{13}=\frac{\nu_{31+\nu_{21} \nu_{32}}}{E_{2} E_{3} \Delta}=\frac{\nu_{13+} \nu_{12} \nu_{23}}{E_{1} E_{2} \Delta}, \\
& C_{22}=\frac{1-\nu_{13} \nu_{31}}{E_{1} E_{3} \Delta}, C_{23}=\frac{\nu_{32+} \nu_{12} \nu_{31}}{E_{1} E_{3} \Delta}=\frac{\nu_{23}+\nu_{21} \nu_{13}}{E_{1} E_{2} \Delta}, \\
& C_{33}=\frac{1-\nu_{12} \nu_{21}}{E_{1} E_{2} \Delta}, \\
& C_{44}=G_{23}, C_{55}=G_{13}, C_{66}=G_{12},
\end{aligned}
$$

where

$$
\Delta=\frac{1-\nu_{12} \nu_{21}-\nu_{23} \nu_{32}-\nu_{31} \nu_{13}-\nu_{21} \nu_{32} \nu_{13}-\nu_{12} \nu_{23} \nu_{31}}{E_{1} E_{2} E_{3}} .
$$


In what follows we consider that our orthotropic material is in an incremental plane strain state relative to the plane $O x_{1} x_{2}$. In this case it is characterized by the two nonvanishing components of the incremental displacement field

$$
u_{1}=u_{1}\left(x_{1}, x_{2}\right), u_{2}=u_{2}\left(x_{1}, x_{2}\right) .
$$

The only nonvanishing components of the incremental nominal stress are

$$
\begin{aligned}
& \vartheta_{11}=\omega_{1111} u_{1,1}+\omega_{1122} u_{2,2}, \quad \vartheta_{21}=\omega_{2112} u_{1,2}+\omega_{2121} u_{2,1} \\
& \vartheta_{12}=\omega_{1212} u_{1,2}+\omega_{1221} u_{2,1},
\end{aligned}
$$

The only incremental equilibrium equations that must be satisfied are

$$
\vartheta_{11,1}+\vartheta_{21,2}=0, \vartheta_{12,1}+\vartheta_{22,2}=0 \text { in } S,
$$

or, equivalently,

$$
\begin{aligned}
& \omega_{1111} u_{1,11}+\omega_{1122} u_{2,21}+\omega_{2112} u_{1,22}+\omega_{2121} u_{2,12}=0 \\
& \omega_{1212} u_{1,21}+\omega_{1221} u_{2,11}+\omega_{2211} u_{1,12}+\omega_{2222} u_{2,22}=0 .
\end{aligned}
$$

The only involved instantaneous elasticities are

$$
\begin{array}{lll}
\omega_{1111}=C_{11}+\sigma, & \omega_{2222}=C_{22}, & \omega_{1122}=\omega_{2211}+C_{12}, \\
\omega_{1212}=\omega_{2121}+C_{66}, & \omega_{1221}=C_{66}+\sigma, & \omega_{2112}=C_{66}, \\
\omega_{3311}=C_{13}, & \omega_{3322}=C_{23}, &
\end{array}
$$

$\sigma$ being the pre-stress acting in direction of $O x_{1}$ axis.

The equilibrium equations can be expressed in the following operatorial form:

$$
P_{11} u_{1}+P_{12} u_{2}=0, \quad P_{12} u_{1}+P_{22} u_{2}=0
$$

with

$$
\begin{aligned}
& P_{11}=\omega_{1111} \frac{\partial^{2}}{\partial x_{1}^{2}}+\omega_{2112} \frac{\partial^{2}}{\partial x_{2}^{2}}, \quad P_{12}=\left(\omega_{1122}+\omega_{2121}\right) \frac{\partial^{2}}{\partial x_{1} \partial x_{2}}, \\
& P_{21}=\left(\omega_{1212}+\omega_{2211}\right) \frac{\partial^{2}}{\partial x_{1} \partial x_{2}}, \quad P_{22}=\omega_{1221} \frac{\partial^{2}}{\partial x_{1}^{2}}+\omega_{2222} \frac{\partial^{2}}{\partial x_{2}^{2}} .
\end{aligned}
$$

We introduce now the independent complex variables

$$
z_{1}=x_{1}+\mu_{1} x_{2}, \quad z_{2}=x_{1}+\mu_{2} x_{2} .
$$

From these relations, we get

$$
\bar{z}_{1}=x_{1}+\bar{\mu}_{1} x_{2}, \quad \bar{z}_{2}=x_{1}+\bar{\mu}_{2} x_{2} .
$$


Since $\mu_{1} \neq \mu_{2}$, we can see now that the differential equation can be expressed in the following equivalent form:

$$
\frac{\partial^{4} \varphi}{\partial z_{1} \partial \bar{z}_{1} \partial z_{2} \partial \bar{z}_{2}}=0
$$

The general solution of this equation is

$$
\varphi=\varphi\left(x_{1}, x_{2}\right)=f_{1}\left(z_{1}\right)+g_{1}\left(\bar{z}_{1}\right)+f_{2}\left(z_{2}\right)+g_{2}\left(\bar{z}_{2}\right)
$$

where $f_{j}=f_{j}\left(z_{j}\right)$ and $g_{j}=g_{j}\left(\bar{z}_{j}\right), j=1,2$, are arbitrary analytic functions of the complex variables $z_{j}$ and $\bar{z}_{j}$, respectively.

We recall now that $\varphi=\varphi\left(x_{1}, x_{2}\right)$ is a real valued function. We must have $g_{j}\left(\bar{z}_{j}\right)=\overline{f_{j}\left(z_{j}\right)}, j=1,2$.

Denoting by $F_{j}^{\prime}\left(z_{j}\right)=\frac{d F_{j}}{d z_{j}}\left(z_{j}\right), j=1,2$, and introducing the functions $\Phi_{j}=\Phi_{j}\left(z_{j}\right), \quad j=1,2$,

$$
\Phi_{j}\left(z_{j}\right)=u_{j} B_{j}\left(\omega_{1122}+\omega_{1212}\right)^{-1} F_{j}^{\prime \prime}\left(z_{j}\right)
$$

with

$$
\begin{aligned}
B_{j} & =\omega_{2222} \omega_{2112} \mu_{j}^{2}+\omega_{1111} \omega_{2222}-\omega_{1122}\left(\omega_{1122}+\omega_{1212}\right) \\
& =-\omega_{1111} \omega_{1221} \mu_{j}^{-2}-\omega_{2112} \omega_{1221}+\omega_{1212}\left(\omega_{1122}+\omega_{1212}\right) .
\end{aligned}
$$

we obtain the representation of the incremental fields by two arbitrary analytic complex potential $\Phi_{j}=\Phi_{j}\left(z_{j}\right), j=1,2$ :

$$
\begin{aligned}
& \vartheta_{22}=2 \operatorname{Re}\left\{\Phi_{1}^{\prime}\left(z_{1}\right)+\Phi_{2}^{\prime}\left(z_{2}\right)\right\}, \quad \vartheta_{21}=-2 \operatorname{Re}\left\{a_{1} \mu_{1} \Phi_{1}^{\prime}\left(z_{1}\right)+a_{2} \mu_{2} \Phi_{2}^{\prime}\left(z_{2}\right)\right\} \\
& \vartheta_{12}=-2 \operatorname{Re}\left\{\mu_{1} \Phi_{1}^{\prime}\left(z_{1}\right)+\mu_{2} \Phi_{2}^{\prime}\left(z_{2}\right)\right\}, \vartheta_{11}=2 \operatorname{Re}\left\{a_{1} \mu_{1}^{2} \Phi_{1}^{\prime}\left(z_{1}\right)+a_{2} \mu_{2}^{2} \Phi_{2}^{\prime}\left(z_{2}\right)\right\} \\
& u_{1}=2 \operatorname{Re}\left\{b_{1} \Phi_{1}\left(z_{1}\right)+b_{2} \Phi_{2}\left(z_{2}\right)\right\}, \quad u_{2}=2 \operatorname{Re}\left\{c_{1} \Phi_{1}\left(z_{1}\right)+c_{2} \Phi_{2}\left(z_{2}\right)\right\}
\end{aligned}
$$

The state of the human bone is a plane state relative to the plane $x_{1} x_{2}$. The involved nominal stresses $\vartheta_{21}$ and $\vartheta_{22}$ must satisfy the following boundary conditions on the two faces of the crack (represented as a cut):

$$
\begin{aligned}
& \vartheta_{21}\left(x_{1}, 0^{+}\right)=\vartheta_{21}\left(x_{1}, 0^{-}\right)=0, \\
& \vartheta_{22}\left(x_{1}, 0^{+}\right)=\vartheta_{22}\left(x_{1}, 0^{-}\right)=-p\left(x_{1}\right), \text { for }\left|x_{1}\right|<a .
\end{aligned}
$$


The incremental displacement, nominal stresses and complex potentials are vanishing at large distances from the crack.

Using the representation of incremental stresses with complex potentials $(20)_{1,2}$, boundary conditions (22) and the theory of Riemann-Hilbert problem, [13], we get the following expressions, ([8], [11]), for the complex potentials $\Psi_{j}\left(z_{j}\right)=\Phi_{j}^{\prime}\left(z_{j}\right), j=1,2$ :

$$
\begin{aligned}
& \Psi_{1}\left(z_{1}\right)=\Phi_{1}^{\prime}\left(z_{1}\right)=-\frac{a_{2} \mu_{2}}{2 \pi \Delta \sqrt{z_{1}^{2}-a^{2}}} \int_{-a}^{a} \frac{p(t) \sqrt{a^{2}-t^{2}}}{t-z_{1}} d t \\
& \Psi_{2}\left(z_{2}\right)=\Phi_{2}^{\prime}\left(z_{2}\right)=\frac{a_{1} \mu_{1}}{2 \pi \Delta \sqrt{z_{2}^{2}-a^{2}}} \int_{-a}^{a} \frac{p(t) \sqrt{a^{2}-t^{2}}}{t-z_{2}} d t .
\end{aligned}
$$

In what follows we assume that $g(t)=g=c t$.

We shall analyze the asymptotical behavior of the fields in the neighborhood of the crack tips. This analysis is important since in this way the relationship between the stresses and the input energy rates, in crack extension may be established.

The incremental fields distribution around the (right) tip can be obtained by letting

$$
x_{1}=a+r \cos \varphi, x_{2}=r \sin \varphi .
$$

In a small neighborhood of the crack tip $x_{1} \approx a, x_{2} \approx 0$ and we get that $z_{1} \approx z_{2} \approx a$. Plemelj's functions may be approximated by

$$
\sqrt{z_{j}^{2}-a^{2}}=\sqrt{2 a r} \chi_{j}(\varphi), \chi_{j}(\varphi)=\sqrt{\cos \varphi+\mu_{j} \sin \varphi}, j=1,2 .
$$

Using (25) in (23) we get the following asymptotic values of the complex potentials:

$$
\Psi_{1}\left(z_{1}\right)=\frac{K_{I}}{2 \sqrt{2 \pi r}} \frac{a_{2} \mu_{2}}{\Delta} \frac{1}{\chi_{1}(\varphi)}, \Psi_{2}\left(z_{2}\right)=-\frac{K_{I}}{2 \sqrt{2 \pi r}} \frac{a_{1} \mu_{1}}{\Delta} \frac{1}{\chi_{2}(\varphi)},
$$

and the following asymptotic values of the incremental fields:

$$
\begin{gathered}
\vartheta_{22}=\frac{K_{I}}{\sqrt{2 \pi r}} \operatorname{Re} \frac{1}{\Delta}\left\{\frac{a_{2} \mu_{2}}{\chi_{1}(\varphi)}-\frac{a_{1} \mu_{1}}{\chi_{2}(\varphi)}\right\}, \\
\vartheta_{21}=-\frac{K_{I}}{\sqrt{2 \pi r}} \operatorname{Re} \frac{a_{1} a_{2} \mu_{1} \mu_{2}}{\Delta}\left\{\frac{1}{\chi_{1}(\varphi)}-\frac{1}{\chi_{2}(\varphi)}\right\}, \\
\vartheta_{12}=-\frac{K_{I}}{\sqrt{2 \pi r}} \operatorname{Re} \frac{\mu_{1} \mu_{2}}{\Delta}\left\{\frac{a_{2}}{\chi_{1}(\varphi)}-\frac{a_{1}}{\chi_{2}(\varphi)}\right\},
\end{gathered}
$$




$$
\begin{gathered}
\vartheta_{11}=\frac{K_{I}}{\sqrt{2 \pi r}} \operatorname{Re} \frac{a_{1} a_{2} \mu_{1} \mu_{2}}{\Delta}\left\{\frac{\mu_{1}}{\chi_{1}(\varphi)}-\frac{\mu_{2}}{\chi_{2}(\varphi)}\right\}, \\
u_{1}=2 \sqrt{\frac{r}{2 \pi}} K_{I} \operatorname{Re} \frac{1}{\Delta}\left\{b_{1} a_{2} \mu_{2} \chi_{1}(\varphi)-b_{2} a_{1} \mu_{1} \chi_{2}(\varphi)\right\}, \\
u_{2}=2 \sqrt{\frac{r}{2 \pi}} K_{I} \operatorname{Re} \frac{1}{\Delta}\left\{c_{1} a_{2} \mu_{2} \chi_{1}(\varphi)-c_{2} a_{1} \mu_{1} \chi_{2}(\varphi)\right\},
\end{gathered}
$$

and $K_{I}=p \sqrt{\pi a}$ represents the stress intensity factor corresponding to the first fracture mode.

\section{Generalized fracture criteria. Crack propagation angle in Iliac Bone}

We start this Section extending Sih's strain energy density criterion (SED) and Erdogan and Sih's maximum tangential stress criterion (MTS) for finding the crack propagation direction in human bones, regarded as pre-stressed orthotropic elastic composites.

We denote by $W$ the involved strain energy density, [15]

$$
\frac{d W}{d V}=\frac{1}{2} \vartheta_{k l} u_{l, k}, k, l=1,2 .
$$

Using Eqs. (26), (27) in (28), we conclude that:

$$
W(r, \varphi)=\frac{S(\varphi)}{r}+\text { a regular part }
$$

$S(\varphi)$ being Sih's incremental strain energy density factor and it is given by the following expression:

$$
S(\varphi)=\frac{K_{I}^{2}}{4 \pi} s_{I}(\varphi)
$$

where

$$
\begin{aligned}
s_{I}(\varphi)= & \operatorname{Re}\left[\frac{\mu_{1} \mu_{2}}{\mu_{2}-\mu_{1}}\left(\frac{\mu_{1}}{\chi_{1}(\varphi)}-\frac{\mu_{2}}{\chi_{2}(\varphi)}\right)\right] \operatorname{Re}\left[\frac{1}{\mu_{2}-\mu_{1}}\left(\frac{\mu_{2} b_{1}}{\chi_{1}(\varphi)}-\frac{\mu_{1} b_{2}}{\chi_{2}(\varphi)}\right)\right] \\
& -\operatorname{Re}\left[\frac{\mu_{1} \mu_{2}}{\mu_{2}-\mu_{1}}\left(\frac{1}{\chi_{1}(\varphi)}-\frac{1}{\chi_{2}(\varphi)}\right)\right] \operatorname{Re}\left[\frac{\mu_{1} \mu_{2}}{\mu_{2}-\mu_{1}}\left(\frac{b_{1}}{\chi_{1}(\varphi)}-\frac{b_{2}}{\chi_{2}(\varphi)}\right)\right] \\
& -\operatorname{Re}\left[\frac{\mu_{1} \mu_{2}}{\mu_{2}-\mu_{1}}\left(\frac{1}{\chi_{1}(\varphi)}-\frac{1}{\chi_{2}(\varphi)}\right)\right] \operatorname{Re}\left[\frac{\mu_{1} \mu_{2}}{\mu_{2}-\mu_{1}}\left(\frac{c_{1} \mu_{2}}{\chi_{1}(\varphi)}-\frac{c_{2} \mu_{1}}{\chi_{2}(\varphi)}\right)\right] \\
& +\operatorname{Re}\left[\frac{1}{\mu_{2}-\mu_{1}}\left(\frac{\mu_{2}}{\chi_{1}(\varphi)}-\frac{\mu_{1}}{\chi_{2}(\varphi)}\right)\right] \operatorname{Re}\left[\frac{1}{\mu_{2}-\mu_{1}}\left(\frac{c_{1}}{\chi_{1}(\varphi)}-\frac{c_{2}}{\chi_{2}(\varphi)}\right)\right] .
\end{aligned}
$$


Strain energy density criterion states the following hypothesis for the extension of cracks in a brittle material under slowly applied plane loads:

- The crack extension starts at its tip in radial direction, for that the strain energy density $S(\varphi)$ has a minimum value.

Erdogan and Sih's maximum tangential stress criterion states the following hypothesis for the extension of cracks in a brittle material under slowly applied plane loads, [9]:

- The crack extension starts at its tip in radial direction.

- The crack extension starts in the plane perpendicular to the direction of greatest tension, i.e.

$$
\vartheta_{\theta \theta}\left(\varphi_{c}\right)=\vartheta_{c}, \quad \frac{\partial \vartheta_{\theta \theta}}{\partial \varphi}\left(\varphi_{c}\right)=0, \quad \frac{\partial^{2} \vartheta_{\theta \theta}}{\partial \varphi}\left(\varphi_{c}\right)<0
$$

where

$$
\vartheta_{\theta \theta}(\varphi)=\sigma_{11} \sin ^{2} \varphi-2 \sigma_{12} \sin \varphi \cos \varphi+\sigma_{22} \cos ^{2} \varphi
$$

These hypotheses imply that the crack will start to propagate in a perpendicular direction to the direction of $\varphi_{c}$ for which $\vartheta_{\theta \theta}$ is maximum.

For $\vartheta_{\theta \theta}(\varphi)$ we have the following representation, [8], [9]:

$$
\begin{aligned}
\vartheta_{\theta \theta}(\varphi)= & \operatorname{Re}\left[\frac{\mu_{1} \mu_{2}}{\mu_{2}-\mu_{1}}\left(\frac{\mu_{1}}{\chi_{1}(\varphi)}-\frac{\mu_{2}}{\chi_{2}(\varphi)}\right)\right] \sin ^{2} \varphi \\
& +2 \operatorname{Re}\left[\frac{\mu_{1} \mu_{2}}{\mu_{2}-\mu_{1}}\left(\frac{1}{\chi_{1}(\varphi)}-\frac{1}{\chi_{2}(\varphi)}\right)\right] \sin \varphi \cos \varphi \\
& +\operatorname{Re}\left[\frac{1}{\mu_{2}-\mu_{1}}\left(\frac{\mu_{2}}{\chi_{1}(\varphi)}-\frac{\mu_{1}}{\chi_{2}(\vartheta)}\right)\right] \cos ^{2} \varphi
\end{aligned}
$$

The present generalized mathematical models for fracture criteria provide a means to find crack propagation angle in a the Iliac human bone regarded as an pre-stressed orthotropic elastic material.

Iliac bone is characterized by the following engineering constants (values in GPa), [4]:

$E_{1}=11.6 ; E_{2}=12.2 ; E_{3}=19.9 ; G_{12}=4, G_{13}=5, G_{23}=5.4, \nu_{12}=0.42 ; \nu_{13}=$ $0.23 ; \nu_{23}=0.23 ; \nu_{21}=0.44 ; \nu_{31}=0.39 ; \nu_{32}=0.38$.

We denote by $\sigma_{\theta \theta}(\varphi)$ tangential stresses $\vartheta_{\theta \theta}(\varphi)$ normalized by parameter $\alpha$, i.e.

$$
\sigma_{\theta \theta}(\varphi)=\alpha \vartheta_{\theta \theta}(\varphi), \text { with } \alpha=\frac{1}{p} \sqrt{\frac{2 r}{a}} .
$$




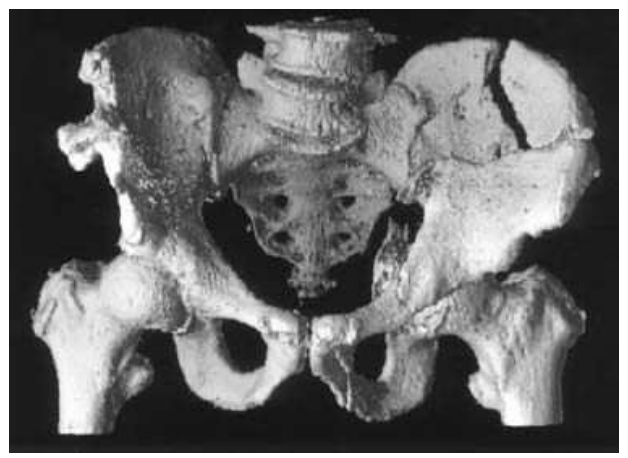

Figure 2: Fracture of the Iliac bone, [18]

In what follows we consider that our initial stress $\sigma \in[-1.5 \mathrm{GPa}, 0]$.

We plotted 3D the normalized incremental strain energy density $s_{I}(\varphi)$ (Fig. 3 left) and $\sigma_{\theta \theta}(\varphi)$ (Fig. 3 right), versus $\varphi \in[-\pi / 2, \pi / 2]$ and $\sigma \in[-1.5 \mathrm{GPa}, 0]$ for Iliac bone.
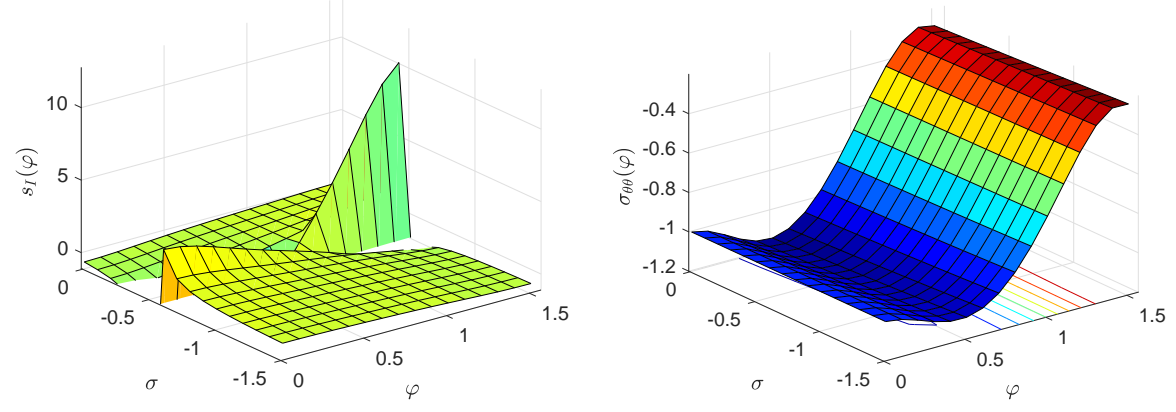

Figure 3: Normalized incremental strain energy density $s_{I}(\varphi)$ (left) and normalized tangential stresses $\sigma_{\theta \theta}(\varphi)$ (right) versus $\varphi$ and $\sigma$

Due to the fact that we are in the case of theory of pre-stressed bodies implying small over great strains we shall consider the case $\sigma \in[-0.5 \mathrm{GPa}, 0]$. So, in this case, from Fig. 3 we observe that function $s_{I}(\varphi)$ (Fig. 3 left) is an increasing function versus $\varphi \in[0, \pi / 2]$, with minimum value for $0^{\circ}$ and using generalized Sih's strain energy density criterion crack will propagate along its line.

The function $\sigma_{\theta \theta}(\varphi)$ (Fig. 3 right) has maximum value in a vicinity of $\pi / 2$ and using generalized maximum tangential stress criterion the crack starts to 
propagate in the plane perpendicular to the direction of $\pi / 2$, i.e. crack will propagate along its line.
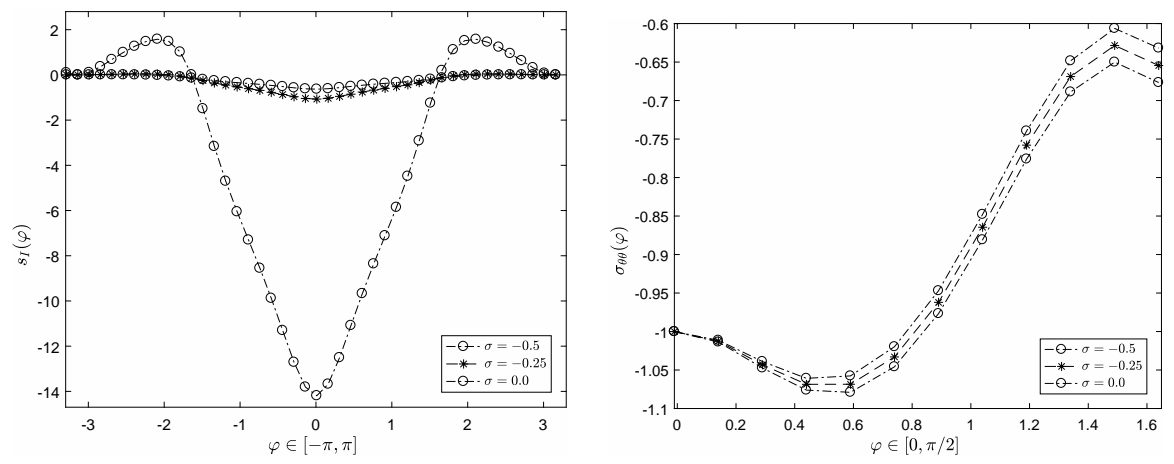

Figure 4: Normalized incremental strain energy density $s_{I}(\varphi)$ (left) and normalized incremental tangential stresses $\sigma_{\theta \theta}(\varphi)$ (right) versus $\varphi$

We observe that in considered case when the pre-stress $\sigma \in[-0.5 \mathrm{GPa}, 0]$, normalized tensile stress $\sigma_{\theta \theta}(\varphi)$ and normalized strain energy density $s_{I}(\varphi)$ are increasing versus $\sigma$. Also, we remark that in the vicinity for $\sigma=-0.5 \mathrm{GPa}$ the function $s_{I}(\varphi)$ changes it convexity. This fact represents an important thing and have to be studied in future researches and validate with numerical or experimental studies.

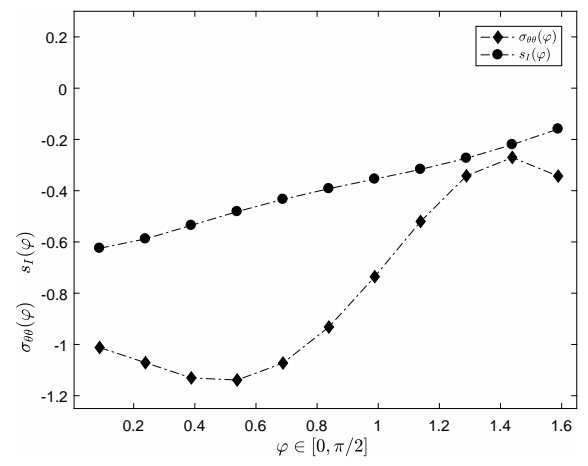

Figure 5: Normalized strain energy density $s_{I}(\varphi)$ and normalized tangential stresses $\sigma_{\theta \theta}(\varphi)$ versus $\varphi$ for un pre-stressed Iliac bone

From Fig. 4, where we plotted normalized tensile stress $\sigma_{\theta \theta}(\varphi)$ and normal- 
ized strain energy density $s_{I}(\varphi)$ versus $\varphi$ for three values of $\sigma \in\{-0.5,-0.25,0\}$, and from Fig. 5, where we plotted normalized tensile stress $\sigma_{\theta \theta}(\varphi)$ and normalized strain energy density $s_{I}(\varphi)$ versus $\varphi$ in the case of un pre-stressed Iliac bone, we observe that in these three particular cases the crack will propagate along its line, too. Both considered criteria furnish us that the crack will propagate along its line.

\section{Conclusions}

Using the theories of Guz's and Muskhelishvili's formalism, we determine the incremental fields in an initially deformed orthotropic elastic composite.

The presented 2D quasistatic mathematical model provides a means to find crack propagation angle for an Mode I crack in Iliac bone regarded as orthotropic materials with initial fields.

In the case of pre-stressed orthotropic elastic composites, we generalized Sih's strain energy density criterion (SED) and Erdogan and Sih's maximum tangential stress criterion (MTS) for finding the crack propagation direction in human bones. Using aforementioned criteria, we found the same result, that a crack in Iliac bone, regarded as a pre-stressed orthotropic elastic composites propagates along its line.

Future numerical results or experimental tests have to confirm or not that our study, in the case of small over great strains, regarding generalization of Sih's criteria is valid, i.e. the crack in pre-stressed in Iliac bone will propagate in horizontal direction.

Also, determination of the critical stress which produces crack propagation and researches regarding the occurrence of resonance phenomena have be taken into account in further studies.

\section{References}

[1] Altenbach, H., Altenbach, J.W. and Kissing, W., Mechanics of Composites Structural Elements, Springer, 2004

[2] Argani, L., Bigoni, D., Capuani, D. and Movchan, N.V., Cones of localized shear strain in incompressible elasticity with prestress: Green's function and integral representations, Proc. R. Soc. A, 470(2014), pp. 01-17

[3] Capuani, D., Bigoni, D. and Brun, M., Integral representations at the boundary for Stokes flow and related symmetric Galerkin formulation, Arch. Mech., 2005, 57(5)(2005), pp. 363-385

[4] Cowin, S.C. et all, Properties of bone in Handbook of Bioengineering, McGrawHill, New York, 1987 
[5] Craciun, E.M., Soos, E., Interaction of two unequal cracks in a prestressed fiber reinforced composite, Int. J. of Fracture 94(1998), pp. 137-159

[6] Craciun, E.M., Soos, E., Crack propagation in the third fracture mode, Rev. Roum. Math. Pures Appl. 45(2000), pp. 229-234

[7] Craciun, E.M., Soos, E., Sih's Generalised Fracture Criterion for Prestressed Orthotropic and Isotropic Materials, Rev. Roum de Sci. Techn.- Mc.Appl. 45, p.663-670

[8] Cristescu, N., Craciun, E.M. and Soos, E., Mechanics of Elastic Composites, CRC Press, Chapman \& Hall, 2004

[9] Erdogan, F., Sih, G.C., On the crack extension in plates under plane loading and transverse shear, ASME, J. Basic. Eng. 85(1963), p. 519-525

[10] Ghita, C., Pop, N. and Popescu, I. N., Existence result of an effective stress for an isotropic visco-plastic composite, Computational Materials Science, 64(2012), pp. 52-56

[11] Guz, A.N., Mechanics of brittle fracture of pre-stressed materials, Visha Shcola, Kiev, 1983

[12] Mishra, P. K., Das, S. and M. Gupta. Interaction between interfacial and sub-interfacial cracks in a composite media. Revisited, ZAMM-Journal of Applied Mathematics and Mechanics/Zeitschrift fr Angewandte Mathematik und Mechanik 96(9)(2016).

[13] Muskhelishvili, N.I., Some Basic Problems of Mathematical Theory of Elasticity, Nordhoff, Groningen, 1953

[14] Radi, E., Bigoni, D. and Capuani, D., Effects of pre-stress on crack-tip fields in elastic, incompressible solids, International Journal of Solids and Structures, 39(2002), pp. 3971-3996

[15] Sih, G.C. A special theory of crack propagation, in: Mechanics of Fracture, vol. I, Editor G.C. Sih, Noordhoof Int. Publ. Leyden, 1973, pp. XXI-XLV

[16] Taylor, W.R., Determination of orthotropic bone elastic constants using FEA and modal analysis, Journal of Biomechanics 35 (2002), pp. 767-773

[17] Zimmermann E.A. et all, Mixed-mode fracture of human cortical bone, Biomaterials, 30 (2009) pp. 5877-5884,

[18] http://radiologykey.com/trauma-4/, Fig. 1.11: 3-D visualization of fractures of left iliac wing and acetabulum.

\footnotetext{
1 "Ovidius" University of Constanta, * Corresponding author: M. Popa

Forensic County Service, 2 Zmeurei Alley, Constanta, Romania

e-mail: mariuspopa_2005@yahoo.com

${ }^{2}$ Technical University of Cluj-Napoca, N.U.C.B.M., Romania
} 